\title{
Comparison of Different Predictive Controllers with Multi-objective Optimization. Application to an Olive Oil Mill
}

\author{
A. Núñez-Reyes*, C.B. Scheffer-Dutra**, C. Bordons*1 \\ *Dpto. de Ingeniería de Sistemas y Automática. Universidad de Sevilla \\ Camino de los Descubrimientos, s/n. 41092 Sevilla, Spain \\ e-mails:\{amparo, bordons\}@cartuja.us.es \\ **Dpto. Automação e Sistemas. Universidade Federal de Santa Catarina \\ UFSC/DAS Caixa Postal 476. Florianópolis-SC-88040 900, Brazil \\ e-mail: cynthia@lcmi.usfc.br
}

\begin{abstract}
This paper presents a comparison of different multiobjective predictive controllers that can be applied to an olive oil mill. The work is focused on the choice of one of the controllers to be implemented in the real plant. Previous studies showed the necessity of including objectives with different priorities in the control scheme. The control strategies have been tested under simulation, showing that good performance can be obtained by the use of a predictive controller with multi-objective prioritization. The work analyzes three different strategies that try to fulfill the specifications, showing their performances in different situations. Implementation issues, such as computational cost, are also considered since this topic is crucial for a successful application on the real plant. The results are illustrated with some simulations based on real data.
\end{abstract}

\section{Introduction}

The automatic control of the extraction of oil out of olives is still an open field, since many installations are usually operated in manual mode. As olive oil mills are becoming bigger the chances for automation are increasing, therefore it is important to acquire the necessary knowledge of the process behaviour in order to design the appropriate control strategies.

Model Predictive Control (MPC) can be considered as the most popular advanced control technique in industry [1], due to its ability to operate the process in such a way that multiple and changing operational criteria (economical, safety, environmental or quality) can be fulfilled in the presence of changes in process characteristics. Although most MPC strategies are based on optimizing a single-cost objective function, in many situations the behaviour of the process cannot be evaluated that way, since there may exist different, and sometimes conflicting, control objectives. The need for the consideration of multiple control objec-

\footnotetext{
${ }^{1}$ This work was supported by FEDER, 1FD97-0836N (Spain) and by CAPES, BEX0704/00-8 (Brazil)
}

tives is due to the fact that processes have to be operated differently when they are at different operating stages and that even if the process is working at a particular operating stage, the control objective may depend on the value of the variables. Furthermore, in many cases, the control objective is not to optimize the sum of the squared errors, but to keep some variables within specified bounds. Notice that this situation is different to the constrained control MPC, as the objective is to keep the variable there, although excursions of the variable outside this region, though not desirable, are permitted. In constrained MPC, the variables should be kept within the prescribed region because of physical limitations, plant safety or other considerations. These types of objectives can be expressed by penalizing the amount by which the offending variable violates the limit. This work applies these ideas to an olive oil mill. This is a multivariable plant which several objectives to be fulfilled, which are also logic-dependent. The process has extra degrees of freedom, which allows different control strategies. The practical experience in the real plant demonstrated that the process can be controlled by a constrained MPC, limiting the manipulated variables. However, the possibility of including objectives with different priorities in the control scheme, could improve the industrial performance. Furthermore, it is important to evaluate the mathematical costs of controller implementation, which can be crucial for the real time application on the plant.

The paper is organized as follows. In section 2 a description of the process is presented. The model identification is described in section 3 . The control strategy applied to the model is described in section 4 , while the implemented controllers are presented in section 5. Section 6 is dedicated to presenting some simulation results and performance analysis of the controllers. Finally, some conclusions are drawn in section 7.

\section{Process Description}

The elaboration of olive oil is achieved by extracting oil out of olives purely by mechanical means, without chemical reactions. All the operations that are performed are aimed at 
extracting the maximum quantity of juice of the raw material without losing quality. In order to do that, the process is composed of several operations: reception of raw material (olives), washing, preparation, extraction, and storage of the produced oil [2]. Figure 1 shows the most important phases of the process.

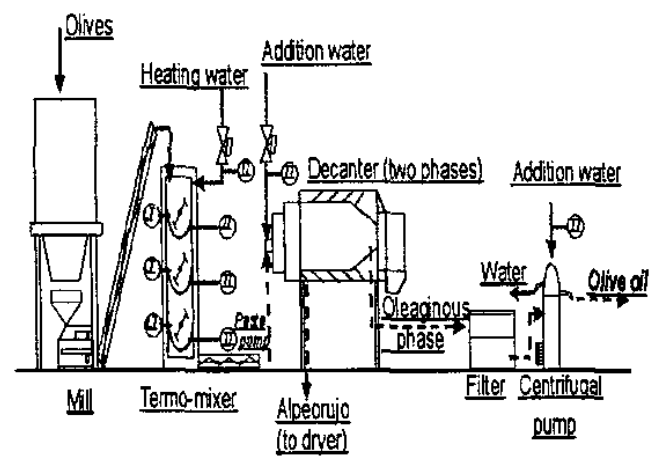

Figure 1: Olive oil mill - process description

The preparation phase consists of two subprocesses. The first one is olive crushing by an special mill, whose objective is to destroy the olive cells where oil is stored. The second one aims at homogenizing the paste by revolving it while its temperature is kept constant at a specified value (around $35^{\circ} \mathrm{C}$ ). This is performed in a machine called thermomixer, which homogenizes the three phases of the paste (oil, water and by-product (alpeorujo)) while exchanging energy with surrounding pipes of hot water. This is done in order to facilitate oil extraction in the mechanical separator. The operation conditions in the thermomixer are really important since they can dramatically affect the quality and quantity of the final product. As a good homogenization is needed, the paste is heated in order to facilitate mixing, since the paste turns more fluent when temperature rises. But there is an upper temperature limit behind which olive oil loses quality (flavour, fragrance, etc.) due to the oxidation process and the loss of volatile components. Therefore, keeping low values of temperature will be a high-priority objective. Experiences of modeling and predictive control of this phase are described in [3]. The next stage is based on the separation of the product phases by means of a centrifuge. This is a continuous process which separates the different components that constitute the paste by means of centrifugal force. This separation is made in the horizontal centrifuge or decanter, that separates olive oil from byproduct. In order to perform a good separation, the paste that enters the decanter must be accommodated. Its flow must be controlled to a setpoint that depends on operating conditions and some water must be added depending on the properties of the raw material. Finally, the last stage of the system consists of the storage and the conservation of the obtained oil. Several factors affect the olive oil extraction process. The final product quality and the industrial yield are influenced by different process variables. Next, the most important variables will be described:

- Temperature in the thermomixer. The heating of the paste has to be constant and gradual since abrupt changes affect negatively the quality of the final product. Two main difficulties appear: the first one is the existence of large delays due to the thermal nature of the process and the second one is caused by the on-off mechanism of feeding the paste.

- Residence time. Another important fact to be considered is the mixing time (residence time) inside the thermomixer. A short time drives to incomplete mixing and a long one can give rise to emulsions, which interfere with the extraction process.

- Paste consistency. The paste consistency gives information about the paste fluidity degree, which is associated to the olive moisture. This value has a great influence in the water that must be added before the paste enters the decanter.

- Paste flow to decanter. The paste flow to decanter and the water/mass proportion determine the maximum industrial yield. The mass flow is adjustable according to the olive type.

- Water flow to decanter. This also determines the extraction effectiveness. The amount of water that is introduced in the decanter must be constant; that is, the sum of the vegetation water of the olive plus the added water must be constant. As is well known, the raw material does not contain a homogeneous moisture, which forces the water flow to be continually adjusted in order to obtain the maximum oil in the decanter.

In the majority of olive oil mills, the process is controlled manually, since there are many factors that affect production. There are many objectives to be fulfilled and the operator must use his experience to have the process under control. This situation justifies the use of a multivariable predictive controller that is able to manipulate several actuators in order to obtain the desired performance. The control strategy applied to this plant is described in section 4.

\section{Model Identification}

Most processes in industry when considering small changes around an operating point can be described by a linear model of, normally, very high order. These models would be difficult to use for control purpose but, fortunately, it is possible to approximate the behaviour of such high order processes by a system with one time constant and a dead time [1]. Therefore, the chosen mathematical structure for the identification of the system is based in the system dynamics of first order with delay.

The input variables and the measurable disturbances have been excited with different steps, in order to identify the system. The parameters of the system model are determined by recursive least squares estimation and the reaction curve method. This model has been validated using real data obtained from an olive-oil mill located in Málaga (Spain). Data was obtained from a series of tests performed in the plant during last years campaign. These data 
have been treated (filtered, sampled, normalized) suitably to reach a acceptable model.

The process matrix fraction description can be seen in equation (1), where the controlled variable $y$ is the oil flow, the manipulated variables $u_{1}, u_{2}$ and $u_{3}$ are, respectively, the temperature in the thermomixer, the paste flow to decanter and the water flow to decanter, and the measurable disturbances $d_{1}$ and $d_{2}$ are related to the olive type. These measurable disturbances have a great influence in the performance, because they represent the olive features for the thermomixer. These measurements have effect on the decision making in the plant control.

$$
[\mathbf{y}]=\left[G_{u_{1}} G_{u_{2}} G_{u_{3}}\right] \mathbf{u}+\left[G_{d_{1}} G_{d_{2}}\right] \mathbf{d}
$$

Notice that the accuracy of the model fitness to real data is good enough for control purposes, despite of its simplicity. Better results could be obtained with higher-order models, but paying the cost of losing simplicity. To get more information about the plant model identification, see [3] and [4].

\section{Control Strategy with Multi-objective Optimization}

The control strategy that has been used to control the olive oil mill can be seen as two control levels, as a cascade structure. A multivariable constrained MPC was implemented to track the oil flow to a desirable reference, modifying the manipulated variables that are the reference signals to an inner loop which operate with classical monovariables controllers PID. The industrial implementation of the MPC has shown the importance of including economical and control objectives in the oil production system [4]. The multi-objective algorithm implemented in the olive oil mill presents four control (and economical) objectives. The control priorities are related to the operator entries in the plant. These entries are called $\mathbf{y}_{o p}$ to the setpoint and $\mathbf{u}_{o p}$ to the desirable manipulated variables. The performance criteria selected in order of decreasing importance are given as follow:

1. keep the thermomixer temperature as near as possible to the optimum value to guarantee the best oil characteristics:

$$
\left|u_{1}-u_{1 \circ p}\right| \leq \epsilon_{u_{1}}
$$

2. maximize the extracted oil:

$$
\left|y-y_{o p}\right| \leq \epsilon_{y}
$$

3. keep the paste flow as close as possible to the operator reference (reduce the necessary flow):

$$
\left|u_{2}-u_{2_{o p}}\right| \leq \epsilon_{u_{2}}
$$

4. reduce the water flow necessary in the production:

$$
\left|u_{3}-u_{3 o p}\right| \leq \epsilon_{u_{3}}
$$

where $\epsilon_{i},\left(i=u_{1}, u_{2}, u_{3}, y\right)$ are very small positive scalars, representing the maximum tolerance admissible to attend the objectives. As can be observed, the control problem includes a "natural" prioritization of objectives. Anyway, three different controllers were implemented in order to solve this problem. These have been implemented in Visual $\mathrm{C}++$ programming language, with the inclusion of the NAG routines in order to solve mixed integer linear programming problems, using a branch an bound method. An integrated platform for the control and automation of the oil mill production has been used, as can be seen in figure 2. This system is made up of different modules connected through a local area network (LAN). These modules are constituted by the software implemented in Visual $\mathrm{C}++$ to support the controllers, a SCADA used to solve the requirements related to data processing and the automation in lower level, and finally a PLC, where the software programmed under SCADA is executed as well as the connection to the real plant is managed.

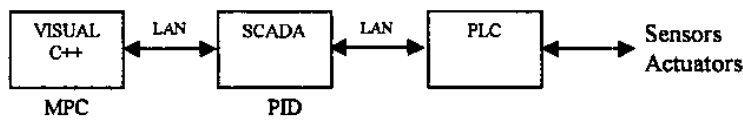

Figure 2: Control Structure

\section{Implemented Multi objective Controllers}

Three different predictive controllers have been compared, all of them trying to fulfill the previous specifications.

The first approach considers each objective to have been transformed into minimizing a quadratic function $J_{i}$, subject to a set of linear constraints on the decision variables $\mathbf{R}_{\mathbf{i}} \mathbf{u} \leq \mathbf{a}_{\boldsymbol{i}}$. The future control sequence can be determined by minimizing the following objective function:

$$
J=\sum_{i=1}^{m} \beta_{i} J_{i}
$$

subject to $\mathbf{R}_{\boldsymbol{i}} \mathbf{u} \leq \mathbf{a}_{i}$; for $i=1, \cdots, m$.

The importance of each of the objectives can be modulated by appropriate setting of all $\beta_{i}$. This is, however, a nontrivial matter in general as it is very difficult to determine the set of weights which will represent the relative importance of the control objectives. Furthermore, practical control objectives are sometime qualitative, making the task of determining the weights even more difficult. This first controller was implemented as a constrained MPC, with the following multi-objective function:

$$
J=\delta\left(y-y_{o p}\right)^{2}+\lambda(\Delta u)^{2}+\gamma\left(u-u_{o p}\right)^{2}
$$

In the sequence, it will be referred as weighted MPC. A possibility to implement the relative importance of the control objectives is to prioritize by giving much higher values to the corresponding weights. That is, the objective of greater priority, in this case to keep the thermomixer temperature as near as possible to the optimum value, must be accomplished before other objectives of less priority are considered. As can be noticed, there are three parameters to tune 
the controller: $\delta, \lambda$ and $\gamma$. However this is a difficult task which is usually done by a trial and error method.

Another possibility was introduced in [5], that presented a way of including multiple prioritized objectives into the model predictive control, combining propositional logic using integer variables with quantitative models. These ideas were extended in [6] using the new mixed logic dynamic (MLD) framework, which allows one to represent systems which can be described by interdependent physical laws, logical rules and operating constraints.

The first objective is the most important one, since the variation of $u_{1}$ can change the final product characteristics. The second objective is the adjustment of output variable to be controlled to its setpoint. The third and the last objectives are related to the energy savings in the production. Each one of these objectives is associate to a distinct $L_{i}, i=1, \cdots, 4$, with different prioritization weights. The design of weights is an arbitrary procedure, guided by the heuristic. In order to apply this procedure to MPC, for each time in the prediction horizon, the prioritization problem is recalculated. The optimum values obtained from the objectives prioritization are used as desirable goal to a MPC controller. The cost function implemented in MPC consists of a weighted sum of squares of the individual objectives, expressed as (6). The method presented in [5] consists of describing the qualitative information in terms of propositional logic and, using integer variables, translate the propositions into linear constraints. In this case considers the process with 4 prioritized control objectives $O_{i}$. Suppose that objective $O_{i}$ has a higher priority than objective $O_{i+1}$ and that the objectives can be expressed as: $\mathbf{R}_{i} \mathbf{u} \leq \mathbf{a}_{i}$. The main idea consists of introducing integer variables $L_{i}$ which take the value one when the corresponding control objective is met and zero otherwise. Objectives are expressed as:

$$
\mathbf{R}_{i} \mathbf{u} \leq \mathbf{a}_{i}+K_{i}\left(1-L_{i}\right)
$$

where $K_{i}$ is a conservative upper bound on $\mathbf{R}_{i} \mathbf{u}-\mathbf{a}_{\boldsymbol{i}}$. If objective $O_{i}$ is satisfied, $L_{i}=1$ and the reformulated objective coincides with the original control objective. By introducing $K_{i}$, the objective (constraint) is always satisfied. The prioritization of objectives can be established by imposing the following constraints:

$$
L_{i}-L_{i+1} \geq 0 \text { for } i=1, \cdots, m-1
$$

To improve the degree of the constraint satisfaction of objectives that cannot be satisfied $O_{f}$, a slack variable $\alpha$ satisfying the following set of constraints is introduced:

$$
\mathbf{R}_{i} \mathbf{u} \leq \mathbf{a}_{i}+\alpha+K_{i}\left[(i-1)+\left(1-L_{i}\right)-\sum_{j=1}^{i-1} L_{j}\right]
$$

and the objective function to be minimized, subject to (7), $(8),(9)$, is:

$$
J=-K_{\alpha} \sum_{i=1}^{m} L_{i}+f(\alpha)
$$

where $f$ is a penalty function of the slack variable $\alpha$ (positive and strictly increasing) and $K_{\alpha}$ is an upper bound on $f$. The optimization algorithm will try to maximize the number of satisfied objectives $\left(L_{i}=1\right)$ before attempting to reduce $f(\alpha)$. More details for this controller are presented in [4]. When the process and the function $f$ selected are linear, the problem of maximizing (10) must be solved by mixed integer linear programming (MILP). The necessity of a great mathematical support to solve the MILP problems must be evaluated in sense to implement on the real plant. This controllers in the following will be referred as prioritized MPC. Using these previous controllers ideas, another approach was implemented. In this case, the issue is to create a control structure that uses a decision list based in a set of if-then statements to select the current objective function that must be supplied to the MPC. The logic implementation is relative to the objectives importance. The objective functions implemented are:

$$
\begin{gathered}
J_{1}=\delta\left(y-y_{o p}\right)^{2}+\lambda(\Delta u)^{2}+\gamma_{1}\left(u-u_{o p}\right)^{2} \\
J_{2}=\delta\left(y-y_{o p}\right)^{2}+\lambda(\Delta u)^{2} \\
J_{3}=\delta\left(y-y_{o p}\right)^{2}+\lambda(\Delta u)^{2}+\gamma_{3}\left(u-u_{o p}\right)^{2} \\
J_{4}=\delta\left(y-y_{o p}\right)^{2}+\lambda(\Delta u)^{2}+\gamma_{4}\left(u-u_{o p}\right)^{2}
\end{gathered}
$$

In each case, the $\delta, \lambda$ parameters are fixed and the value of $\gamma$ changes according to the desirable levels of priorities. The change for one of these functions will depend of the excursions of the variables outside a bounded region, selected by the algorithm. This controller will be referred as structured MPC.

\section{Simulation Results and Performance Analysis}

To illustrate the application of these approaches, consider the olive oil mill described in the section II. The controller uses three manipulated variables, thermomixer temperature, paste flow and water flow, to adjust the control variable, oil flow. The control priorities are implemented as described in section IV. The main idea of the implemented controllers is to fulfill the most possible priorities objectives, besides to obtain good behavior. In order to compare them, the controllers have been submitted to reference changes and steps in the three inputs as well as different disturbances. In all cases, the dotted lines correspond to the weighted MPC signals, the thick and thin solid lines to the prioritized MPC and the structured MPC, respectively. The figures appear by priority order, that means, show the values of $u_{1}$, the output $y, u_{2}$ and $u_{3}$, in sequence. In the inputs graphics, the dashed lines refer to the desirable inputs, as well as in the output graphic, this curve corresponds to the reference. For all simulations, the values assumed by the variables are normalized.

It is considered that a controller fulfills an objective when the value of the corresponding signal does not exceed a band given with respect to the desirable values. The values of these bands have been taken according to the experience in the real plant.

For the first simulation, showed in the figure 3, a $20 \%$ step change in $u_{1}$ has been applied. It is possible to observe how the first objective is fulfilled by the three simulated controllers, reaching the value of $u_{1}$, the desirable reference. The second objective is also reached by all the controllers, 
although the behavior of prioritized MPC is much smoother that the others, arriving at the reference before. The third objective is only handled by the prioritized MPC controller. Whereas the others violate the reference of $u_{3}$, note that structured MPC presents a smaller error than the weighted MPC, although it does not fulfill this objective. And finally, the fourth objective is not reached by any of the three controllers. Notice that the error of prioritized MPC is superior to the others in the objective of smaller priority, because sacrifices $u_{3}$ to reach the objectives of greater priority.
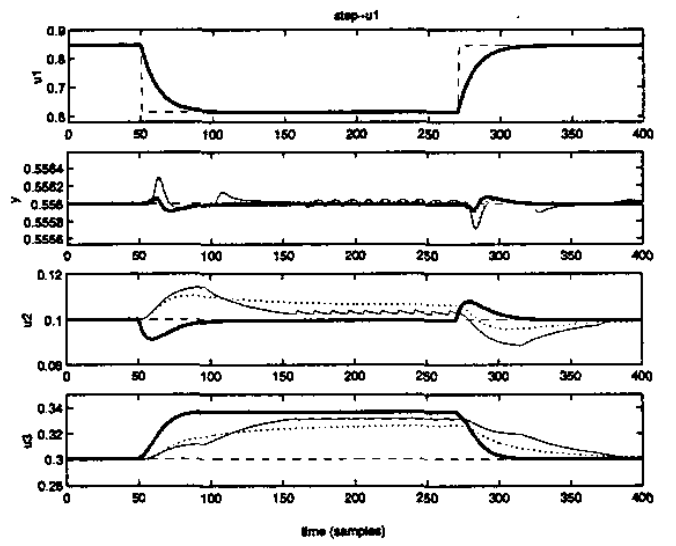

Figure 3: System response to a $20 \%$ step change in $u_{1}$

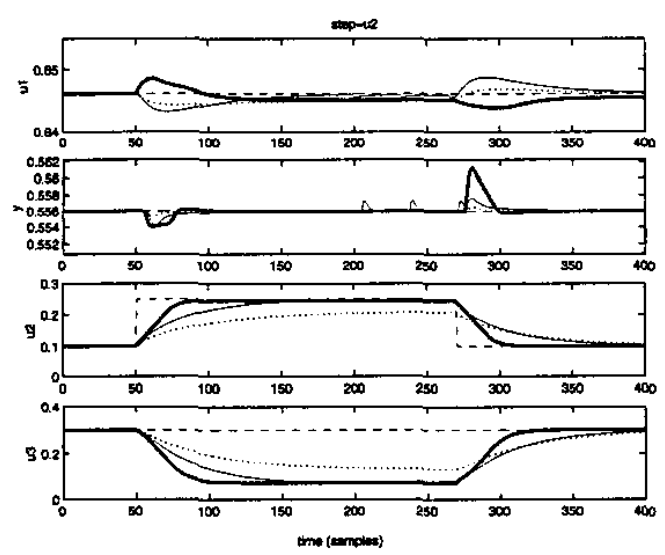

Figure 4: System response to a $14 \%$ step change in $u_{2}$

In figure 4 , the controllers behavior is shown when a $14 \%$ change in $u_{2}$ produced by the operator has been applied. The prioritized MPC and structured MPC controllers fulfill all the objectives except the last one, whereas the weighted MPC just turns the first and second ones. It is necessary to emphasize that prioritized MPC moves away, in the objectives of greater priority, from the desirable values, without leaving the allowed security bands, thus it is able to reach one objective more. This is possible because the design of prioritized MPC includes a parameter in its tuning, denominated comfort, which is easily tuned according to the security bands.

In the third case, figure 5 , a $33.4 \%$ step in the input $u_{3}$ is produced. The three controllers reach the first and the second objectives, but the prioritized MPC and structured MPC controllers are closer of the second objective reference than weighted MPC. For this, they move away much more in the smaller priority objectives.

In the fourth simulation, figure 6 , a $20 \%$ setpoint change in the oil flow has been executed. The prioritized MPC and structured MPC controllers behave better than weighted MPC, since they reach all the objectives, unless the one of smaller priority. The weighted MPC generates a worse behavior, since it fulfills only the two first priorities objectives.

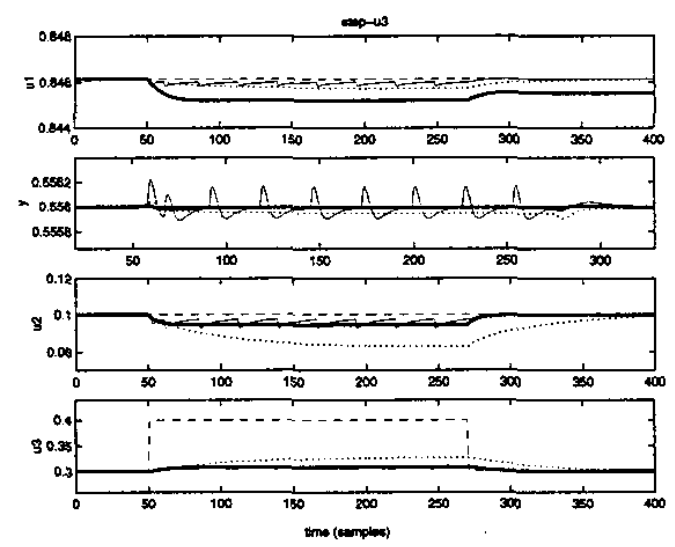

Figure 5: System response to a $33.4 \%$ step change in $u_{3}$

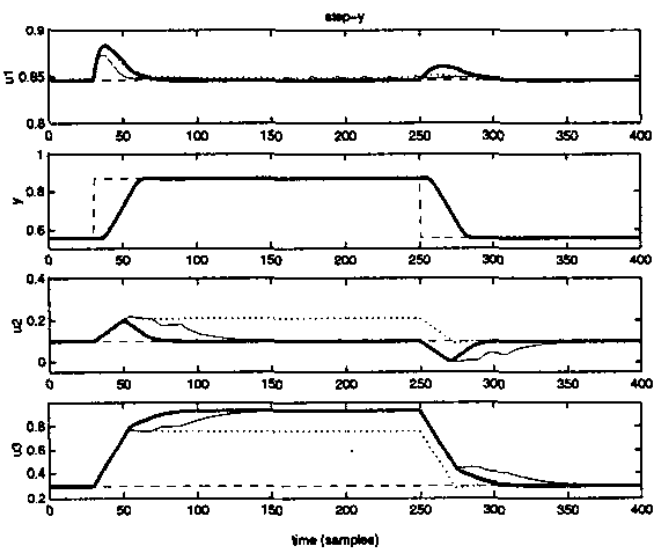

Figure 6: System response to a $20 \%$ step change in $y$

In order to obtain a quantitative comparison of the objectives violation, a behavior index ITSE, see equation (15), was used to analyze the error in the input as a difference between the desirable variables input and their real values, and in the case of the output as a difference between the real output and the reference.

$$
i t s e=\int_{0}^{T} t \cdot e^{2} d t
$$


In order to compare the three controllers in the four previous situations, an index table has been calculated, considering a weighted average of the ITSE values, according to the priority of each objective, as a new index:

$$
\text { witse }=\sum_{i=1}^{4} W_{i} * i t s e_{i}
$$

The following table results of the errors calculation for each one of the previous simulations, referred as $u_{1}, u_{2}, u_{3}$ and $y$ changes, in this order. In table 1 , it is observed that for all

\begin{tabular}{|c|c|c|c|}
\hline Control & Weighted & Structured & Prioritized \\
\hline$u_{1}$ & 0.025118 & 0.005683 & 0.008618 \\
$u_{2}$ & 7.402732 & 6.729413 & 4.062447 \\
$u_{3}$ & 0.355468 & 0.005420 & 0.012871 \\
$y$ & 11.953248 & 8.243687 & 2.811858 \\
\hline
\end{tabular}

Table 1: WITSE Indexes

situations the errors values of the prioritized MPC controller are inferior to the other controllers errors. As well, the structured MPC controller presents smaller errors than the weighted MPC. Using the ITSE index, it is possible to demonstrate that the behavior of the weighted MPC is worse than the others. The prioritized MPC and the structured MPC operation are very similar and extremely dependent of the pararneters tuning. This is the reason why it is difficult to generalize the goodness of each one, since this will depend on the nature of the system to be controlled. If the application needs a controller who responds smoothly in case of changes in the inputs and the reference, it is necessary to tune the $\gamma$ parameter of the structured MPC with smaller values than those of the prioritized MPC controller. This tuning results an increase of the errors index of the structured MPC. In contrast, if a system requires fast responses and do not concern about the abruptness in the controller signals, the structured MPC can be tuned to get ISE index smaller than prioritized MPC.

All the controllers presented in this article are perfectly valid to be implement in real plants, but the selection between one or another controller will be made based on the advantages and disadvantages of each one, on the application resources and coverall on the exigencies and requirements of the control system.

The prioritized MPC controller, apart from having the best behavior with respect the fulfilment to the priorities of the objectives, has a great advantage in front to the weighted MPC, since it does not need to tune the weights of the objective function $(\gamma, \lambda, \delta)$. This is one of the contributions of this controller since the tuning of these weights costs much time and is not simple, because of the system complexity: a multivariable, slow and with great delays process. However, this controller has other tuning parameters that are called objectives comforts, which are much more easy and intuitive to fit. The main disadvantage of the prioritized MPC is the necessity of an important additional cost, from the computacional and economic point of view, Since it requires a software package that provides solution to the MILP problem. Other disadvantage is the feasibility management necessary in case that the MILP problem be unfeasible.
The structured MPC controller was thought to resolve the disadvantages of prioritized MPC. This controller either does not need to tune the weights of the cost function, and its time of calculation is minor than the other controllers. Its behavior respect to fulfill the priorities of the objectives is quite good, although it is generally inferior to the prioritized MPC. Its main inconvenience consists of reaching a balance between the different objective functions, to avoid abrupt signals when switching.

\section{Conclusions}

This paper has carried out the analysis and the comparison of the behaviour of three multi-objective controllers with priorities, applied to the control of an olive oil mill. The outcome of the study has been that although all control strategies can be successfully applied to the system, each one has different advantages and drawbacks.

Weighted MPC controller shows worse behaviour versus the rest of controllers due to the lower number of objectives that can be reached. The need of tuning the weights of the cost function is another disadvantage to be added to this controller. However, it does not need additional software for its development. With the prioritized MPC, the highest number of satisfied objectives, without the need of tuning the weights, is guaranteed. The weak point is the requirement of using additional and complex software, having as a consequence an increasing in the whole cost of the system and the processing time.

An intermediate option is the use of the structured MPC controller. It does not require weights in the objective function but, on the other hand, it shows abrupt behaviour in the switching between different objective functions. A good alternative to undertake the control of the system could be a controller that comprise the structured MPC and prioritized MPC, in addition of a feasibility handler. Hence, the structured controller would replace the prioritized MPC in case of unfeasible solutions.

\section{References}

[1] E.F. Camacho and C. Bordons, Model Predictive Controh Springer Verlag, 1999.

[2] L. Civantos, Obtención del Aceite de Oliva Virgen (in spanish), Ed. Agrícola Española, S.A. 1999.

[3] C. Bordons and J.R. Cueli, "Modeling and predictive control of an clive cil mill", in European Control Conference, Porto 2001.

[4] C.B. Scheffer-Dutra, A. Núñez-Reyes and C. Bordons, "Predictive control of an olive oil mill with multiobjective prioritization", in to appear in the Proc. of the 15th IFAC World Congress, Barcelona, July 2002.

[5] M. L. Tyler and M. Morari, "Propositional logic in control and monitoring problems", Automatica, vol. 35, pp. 565-582, 1999.

[6] A. Bemporad and M. Morari, "Control of systems integrating logic, dynamics and constraints", Automatica, vol. 35, pp. 407-427 1999. 